\title{
Intraobserver variation in Doppler ultrasound assessment of pulmonary artery pressure
}

\author{
N V Subhedar, N J Shaw
}

\begin{abstract}
Intraobserver variation associated with the non-invasive assessment of pulmonary artery pressure (PAP), using measurement of pulmonary artery Doppler derived systolic time intervals, was investigated. Forty pairs of independent ultrasound examinations of the pulmonary artery were performed by a single observer in 20 preterm infants, median gestation 27 weeks (range 24-31 weeks). Median age at study was 17 days (range 1-47 days). Paired measurements of acceleration time (AT), ratio between acceleration time and right ventricular ejection time (AT:RVET), corrected AT, and corrected AT:RVET were compared to assess intraobserver agreement.

For the corrected AT:RVET ratio, the mean percentage difference between observations was $-0.9 \%$ (95\% confidence intervals -5.0 to $3.1 \%$ ). The limits of agreement for the two measurements were -26.3 to $24.5 \%$. The coefficient of repeatability was $\mathbf{2 5 . 4 \%}$. Variation for other indices was similar.
\end{abstract}

Non-invasive assessment of PAP using Doppler derived systolic time intervals is associated with considerable intraobserver variation.

(Arch Dis Child 1996;75:F59-F61)

Keywords:pulmonary artery pressure, Doppler ultrasound, intraobserver variation, AT:RVET ratio.

Pulmonary artery Doppler ultrasound is being used increasingly to follow changes in pulmonary artery pressure (PAP) in the neonatal period and beyond. PAP has been assessed non-invasively using right ventricular systolic time intervals in healthy term and preterm infants, ${ }^{1}$ and those with cardiorespiratory disease. ${ }^{2}$ Various indices of pulmonary flow velocity correlate with PAP measured directly at cardiac catheterisation in infants and older children. ${ }^{45}$ Time intervals most commonly used include the acceleration time (AT) and the acceleration time to right ventricular ejection time (AT:RVET) ratio. Further correction for variation in heart rate may be made to give the corrected acceleration time (AT(c)) and corrected acceleration time to right ventricular ejection time ratio (AT:RVET(c)).

An accurate and reproducible non-invasive method of assessing PAP would allow the role of pulmonary hypertension in respiratory disease and the effects of pulmonary vasodilator treatment to be studied in greater detail.
Although the reproducibility of other Doppler ultrasound measurements has been investigated, ${ }^{67}$ there are, to our knowledge, no published studies assessing the variation associated with pulmonary artery Doppler measurements. The aim of this study was to determine the intraobserver variation inherent in using this technique to assess PAP in the newborn period.

\section{Methods}

Preterm neonates were studied during either the acute or recovery phase of respiratory distress syndrome (RDS). Doppler examination of the pulmonary artery was performed using a duplex Doppler ultrasound system (Advanced Technical Laboratories, Ultramark IV) incorporating a $7.5 \mathrm{MHz}$ multifrequency imaging transducer combined with a $5 \mathrm{MHz}$ pulsed wave Doppler transducer. A real time two dimensional image of the main pulmonary artery was obtained using either a parasternal short axis, or a modified long axis view. The sample volume was placed in the centre of the artery just distal to the pulmonary valve leaflets. Before the Doppler signal was recorded, further manipulation of the transducer was necessary to align properly the ultrasound beam to ensure the angle of insonation was below $20^{\circ}$ and to obtain an optimal flow velocity pattern. Using a sweep speed of 100 $\mathrm{mm} /$ second, between three and five consecutive systolic waveforms with clearly defined spectral envelopes were selected for measurement of time intervals.

The integrated electronic calipers of the system were used to measure time intervals directly from the frozen ultrasound image on the screen. The $R-R$ interval was measured from a simultaneous ECG trace displayed on the screen. AT was defined as the interval between onset of ejection and time of peak velocity. RVET was the interval between onset and cessation of systolic blood flow. AT:RVET was the ratio between these two intervals. Correction for heart rate was performed by dividing AT and AT:RVET by the square root of the $R-R$ interval to give $A T(c)$ and AT/RVET(c), respectively.

One or more pairs of examinations were performed on each infant studied by a single observer (NVS). The initial measurement was made "blind" by obscuring the measurement obtained on the screen and printing a hard copy for subsequent analysis. This was immediately followed by a repeat examination on the same baby. Satisfactory Doppler signals were obtained in all infants studied and right 


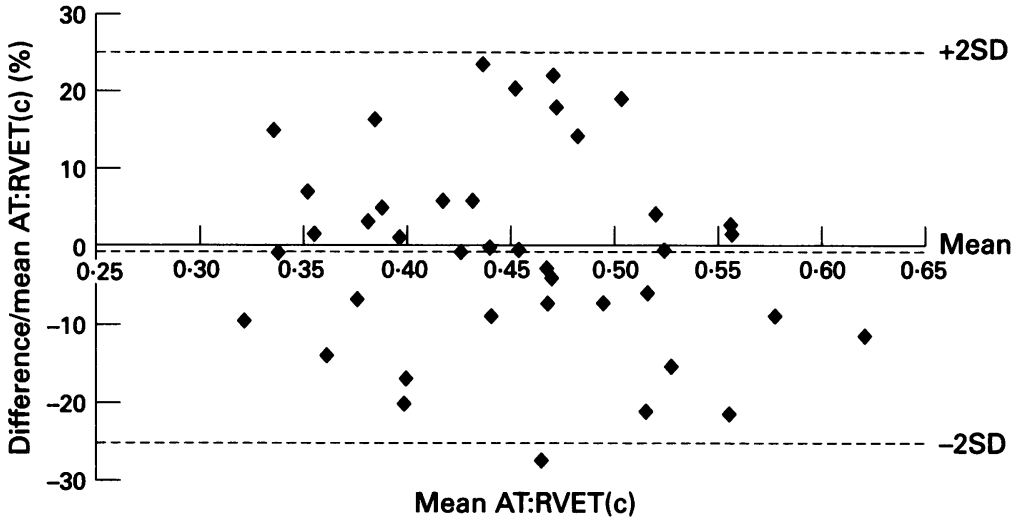

Figure 1 Intraobserver agreement between paired measurements of AT:RVET(c).

ventricular function, as assessed subjectively by two dimensional echocardiography, was normal in all cases. All infants had stable oxygen saturations throughout the study.

Data from paired observations was analysed using the method described by Bland and Altman. ${ }^{8}$ Intraobserver agreement was assessed by plotting the difference between each pair of measurements (expressed as a percentage of their mean value) against the mean value itself.

The study was approved by the local paediatric research ethics committee and informed parental consent was obtained.

\section{Results}

Forty pairs of measurements were made in 20 infants studied. Their median birthweight was 897 (range 680-750) g and gestation 27 (range 24-31) completed weeks. Median age at the time of study was 17 (range 1-47) days. Thirteen infants were receiving assisted ventilation and a further two infants were receiving only supplemental oxygen.

A Bland-Altman plot of the 40 pairs of measurements for the AT:RVET(c) is shown (fig 1). The overall mean percentage difference between each pair of measurements of AT:RVET(c) was $-0.9 \%$ (95\% confidence intervals -5.0 to $+3.1 \%$ ), suggesting that there was no overall systematic bias between paired measurements. The limits of agreement, which included all values between plus and minus 2 SD from the mean, were -26.3 to $+24.5 \%$. The coefficient of repeatability ${ }^{8}$ was $25.4 \%$. Values for the other time intervals were similar (table 1).

\section{Discussion}

Pulmonary artery pressure may be assessed non-invasively using Doppler ultrasonography using one of three methods. Measurement of the maximal velocity of the tricuspid regurgitant jet and subsequent application of the modified Bernoulli equation, ${ }^{9}$ or analysis of

Table 1 Analysis of differences betwen paired measurements of time intervals (\%)

\begin{tabular}{llllll}
\hline & $\begin{array}{l}\text { Overall } \\
\text { mean } \\
\text { difference }\end{array}$ & $\begin{array}{l}\text { Range of observed } \\
\text { differences }\end{array}$ & $S D$ & $\begin{array}{l}\text { Limits of } \\
\text { agreement }\end{array}$ & $\begin{array}{l}\text { Coefficient } \\
\text { of } \\
\text { repeatability }\end{array}$ \\
\hline AT & -1.2 & -26.1 to 29.3 & 12.8 & -26.8 to 24.4 & 25.6 \\
AT(c) & -0.7 & -28.1 to 30.0 & 12.5 & -25.7 to 24.3 & 25.0 \\
AT:RVET & -2.1 & -24.8 to 21.6 & 12.5 & -27.1 to 22.9 & 25.0 \\
AT:RVET(c) & -0.9 & -27.5 to 22.9 & 12.7 & -26.3 to 24.5 & 25.4 \\
\hline
\end{tabular}

maximal ductal flow velocity in a similar way, ${ }^{10}$ both permit quantification of PAP. Pulmonary artery systolic time intervals are more widely applicable in neonatal practice, because the technique of pulmonary artery Doppler ultrasound is more straightforward and satisfactory recordings can be obtained in almost all infants by anyone familiar with using a conventional ultrasound scanner. Such measurements from the pulmonary artery velocity waveform and their derived ratios cannot be used to determine an absolute value of PAP.

This study shows that there is an intraobserver variation when using Doppler derived pulmonary artery systolic time intervals which we believe is clinically important. The method described by Bland and Altman permits quantification of the degree of agreement between two measurements of the same variable. The limits of agreement for independent, paired observations of AT:RVET(c) were between -26.3 and $+25.4 \%$, suggesting that the estimated true value would most probably lie between plus and minus approximately $25 \%$ of any calculated value of AT:RVET(c). For a calculated value of 0.4 , the true value would lie between 0.3 and 0.5 . Therefore, we suggest that repeated measurements be made to help confirm an isolated abnormal value. Furthermore, expression of AT:RVET(c) and other time intervals to three decimal places, as is generally quoted, is inconsistent with the degree of intraobserver variation found.

There are several potential sources of error that may have been responsible for the degree of variation seen in this study. Errors in measurement of time intervals probably accounted for a major part of the total variation. In particular, there was uncertainty in deciding precisely when the velocity waveform left the baseline, reached its peak velocity, and returned to the baseline. These inaccuracies were most important when measuring short time intervals, such as AT, which lasted only between 25 and $60 \mathrm{~ms}$. As the overall intraobserver variation associated with measurement of AT was comparable with other indices, however, this error was probably balanced by the need to make only one measurement, whereas calculation of AT:RVET and AT:RVET(c) involved two or three separate measurements, respectively.

The shape of the pulmonary artery waveform is influenced by both the position of the sample volume and the alignment of the ultrasound beam within the main pulmonary artery. ${ }^{11}$ Two dimensional imaging only allows for consistent positioning of the sample volume and beam in one plane, and therefore small differences in position or direction may have affected the shape of the waveforms, and hence the measurements obtained.

The effect of beat to beat variations in stroke volume, which reflect changes with respiration, may be minimised by using the mean from a greater number of waveforms than was used in this study. Our own observations (unpublished) have shown that the coefficient of variation for AT:RVET(c) decreases with increasing waveforms used (from $14.7 \%$ with three wave- 
forms to $12.3 \%$ with 20 waveforms). Although the limitations of our system prevented us from doing so, calculating the mean ratio from five to 10 waveforms would seem to be a reasonable compromise between improved accuracy and practical acceptability.

Despite the two independent measurements being made within a few minutes of each other, there might have been a change in PAP, although all infants were haemodynamically stable during the study period. Short term variation is common in other cardiovascular parameters, ${ }^{12}$ and similar changes in PAP would have decreased the reproducibility of any method. But as there was no overall systematic difference between observations (the mean observed percentage difference between paired measurements being $-0.9 \%$ for AT:RVET(c)), there does not seem to have been any consistent effect from the procedure itself.

The reproducibilty of other Doppler measurements has been studied, although there are no published studies of the intraobserver variation associated with other non-invasive methods of estimating PAP. Although a previous study seemed to show good agreement between pairs of measurements of AT:RVET (with a correlation coefficient of 0.92 ), ${ }^{1}$ only a single examination was performed on each infant with two observers measuring the AT:RVET independently from one hard copy. Our study is fundamentally different and represents the overall variation present when two independent examinations are made by one observer. We have presented our data expressing the difference between pairs of measurements as a percentage of their mean value in order to describe the degree of agreement uniformly across a range of measurements. This allows comparisons to be made of the variation of different methods, rather than merely expressing variation in absolute figures. Direct comparison with other Doppler studies ${ }^{67}$ is difficult because different methods have been used to quantify the variation present. Nevertheless, our results are in keeping with the variation observed in these studies.

Recent studies using pulmonary artery Doppler ultrasound have described longitudinal changes in groups of infants with RDS or chronic lung disease of prematurity and have shown trends in non-invasively assessed PAP with time. ${ }^{213}$ Despite the inherent intraobserver variation, these authors were still able to demonstrate significant temporal changes. The wide range of values obtained, however, may reflect the degree of variation present. It may be necessary to study a large number of infants to detect small (but perhaps clinically important) differences in Doppler derived time intervals and their ratios.

In conclusion, we have shown that there is considerable intraobserver variation when using Doppler derived systolic time intervals to estimate PAP non-invasively in the neonatal period. As interobserver variation would inevitably be greater still, we suggest that a single observer should make serial measurements in any individual infant. Two consecutive measurements would need to differ by at least $25 \%$ to ensure that a true change in PAP had occurred. Repeated measurements would be helpful to confirm any calculated value, to maximise the reliability of this technique. Although pulmonary artery Doppler measurements offer distinct advantages over other methods of assessing PAP, awareness of these limitations is important.

NVS was supported by the British Heart Foundation (R H Martin Junior Research Fellowship).

1 Evans NJ, Archer LNJ. Postnatal circulatory adaptation in healthy term and preterm neonates. Arch Dis Child 1990;65:24-6

2 Gill AB, Weindling AM. Raised pulmonary artery pressure in very low birthweight infants requiring supplemental in very low birthweight infants requiring supplemental oxygen at 36

3 Fitzgerald D, Evans N, Van Asperen P, Henderson-Smart D. Subclinical persisting pulmonary hypertension in chronic neonatal lung disease. Arch Dis Child 1994;70:F118-22.

4 Akiba T, Yoshikawa M, Otaki S, Kobayashi Y, Nakasato M Suzuki $\mathrm{H}$. Prediction of peak pulmonary artery pressure by continuous wave Doppler echocardiography in infants and children. Pediatr Cardiol 1988;9:225-9.

5 Kosturakis D, Goldberg SJ, Allen HD, Loeber C. Doppler echocardiographic prediction of pulmonary arterial hypertension in congenital heart disease. $A m \mathcal{f}$ Cardiol 1984;53:1110-15.

6 Hudson I, Houston A, Aitchson T, Holland B, Turner T. Reproducibility of measurements of cardiac output in newborn infants by Doppler ultrasound. Arch Dis Child 1990;65:15-19.

7 Moorthy B, Colditz PR, Ives KN, Rees DG, Van't Hoff WG, Hope PL. Reproducibilty of cerebral artery Doppler measurements. Arch Dis Child 1990;65:700-1.

8 Bland JM, Altman DG. Statistical methods for assessing agreement between two methods of clinical measurement. Lancet 1986;i:307-10.

9 Yock PG, Popp RL. Noninvasive estimation of right ventricular systolic pressure by Doppler ultrasound in patients with tricuspid regurgitation. Circulation 1984 70:657-62.

10 Musewe NN, Poppe D, Smallhorn JF, et al. Doppler echocardiographic measurement of pulmonary artery pressure from ductal Doppler velocities in the newborn. $f$ pressure from ductal Doppler veloc

11 Panidis IP, Ross J, Mintz GS. Effect of sampling site on assessment of pulmonary artery blood flow by Doppler assessment of pulmonary artery blood flow by

12 Anthony MY, Evans DH, Levene MI. Cyclical variations in cerebral blood flow velocity. Arch Dis Child 1991;66:12-16.

13 Evans NJ, Archer LNJ. Doppler assessment of pulmonary artery pressure and extrapulmonary shunting in the acute phase of hyaline membrane disease. Arch Dis Child 1991;66:6-11. 